\title{
Does a Low-wall Coverage Stent Have a Flow Diverting Effect in Small Aneurysms?
}

\author{
Hairi Liu, MD'.2, Jooae Choe, MD', Seung Chul Jung, MD', Yunsun Song, MD', Ku Hyun Yang, MD', \\ Kye Jin Park, MD', Hae Won Goo, MD', Won Hyong Park, MD', Dae Chul Suh, MD'
}

Backbround and Purpose: The flow diverting effect of a low-wall coverage stent remains controversial. We evaluted patients who underwent stenting for small aneurysms with a low but potential risk of growth and reviewed related literature.

Materials and Methods: We evaluated 9 small aneurysms among 19 unruptured intracranial aneurysms from eight patients who underwent stenting. The patients had unexplainable severe headache $(n=$ $8)$, aneurysm originating from the anterior choroidal artery $(n=3)$, potential growth or rupture risks including hypertension $(n=5)$, and multiple aneurysms $(n=6)$. Stents with a relatively low-wall coverage ratio (8-10\%) were used. Clinical and angiographic outcomes were assessed.

Results: One $(n=8)$ or two stents $(n=1)$ were used without any procedural difficulties or complications. Although no immediate changes of aneurysm morphology were observed, aneurysms decreased in size $(n=8)$ when examined by DSA $(n=8)$ or MRA $(n=1)$ during a median 28.9-month follow-up. There were no adverse events, including thromboembolism, aneurysm rupture, or stent movement during a median 31.9-month clinical follow-up (range: 17-69 months).

Conclusion: Although a variable degree of aneurysm size decrease may not prevent further growth or rupture of small aneurysms, stenting with a low-wall coverage ratio may have some advantageous hemodynamic effect. Flow modification of stent architecture vs. aneurysm characteristics, including size and location, on long-term outcome, requires further clarification.

Key Words : Stent; Intracranial aneurysm; Hemodynamics

'Departments of Radiology and Research Institute of Radiology, University of Ulsan, College of Medicine, Asan Medical Center, Seoul, Korea 2Department of Interventional Radiology, Taizhou People's Hospital, Taizhou, Jiangsu Province 225300, P. R. China

Received June 22, 2015;

accepted after revision June 27, 2015.

Correspondence to: Dae Chul Suh, MD, Department of Radiology, University of Ulsan, College of Medicine, Asan Medical Center, 88, Olympic-ro 43-gil, Songpa-gu, Seoul 138-736, Korea.

Tel. 82.2.3010.4366 Fax. 82.2.476.0090

E-mail: dcsuh@amc.seoul.kr

This is an Open Access article distributed under the terms of the Creative Commons Attribution Non-Commercial License (http://creativecommons.org/licenses/by-nc/3.0) which permits unrestricted non-commercial use, distribution, and reproduction in any medium, provided the original work is properly cited. 
The flow diverting effect of a low-wall coverage stent remains controversial. Although stenting can lead to improvements in the hemodynamic parameters of aneurysms [1], there are few clinical reports regarding long-term outcomes after stenting $[2,3]$.

A previous hemodynamic study revealed that stenting is more effective for small aneurysms than for large aneurysms because greater improvements in hemodynamic parameters are observed with small aneurysms [4]. Endothelial growth along the stent in the parent artery of the aneurysm may induce significant neck narrowing and cause an obvious decrease in intraaneurysmal flow. Factors including aneurysmal circulation disconnection, aneurysmal in-jet redirection, and formation of a scaffold useful for vessel repair may accentuate aneurysm thrombosis and vessel-wall remodeling $[5,6]$.

We present our results on the management of small, unruptured intracranial aneurysms (UIAs; maximum dimension less than $4 \mathrm{~mm}$ ) by stenting of the parent artery and assess the effects of stenting on long-term outcome.

\section{MATERIALS AND METHODS}

Between July 2006 and October 2013, nine patients with 19 UIAs ( $\mathrm{M}: \mathrm{F}=3: 5$ ) who ranged in age from 24 to 70 years (mean, 51.7 years) were included from prospectively collected data obtained at our institution. Informed consent was obtained from each patient or their relative. This study was approved by our Institutional Review Board.

Symptoms that could not otherwise be explained by clinical examinations or MRI studies of the brain, including headache, dizziness, ocular pain $(n=8)$, hypertension $(\mathrm{n}=5)$ and a family history of aneurysmal rupture $(\mathrm{n}=1)$, were noted (Table 1$)$. One patient with familial polycystic kidney disease who presented with a headache underwent stenting as a result of failed coiling and the potential risk of growth or rupture $(n=$ 1) of an aneurysm. The size of the aneurysms ranged 2.0 to $3.9 \mathrm{~mm}$ (mean $2.9 \mathrm{~mm}$ ) in the maximum length (Fig. 1). Further comments whether stenting can be indicated in these small aneurysms or not is not within the scope of this study. Based on the definition of a wide-neck aneurysm having a dome-to-neck ratio $<2$, two were narrow-neck aneurysms and seven were wide-neck aneurysms (dome-to-neck ratio; range 0.82.1, mean 1.5) among the nine aneurysms.

All UIAs were primarily identified by routine examinations using CTA or MRA. A final diagnosis

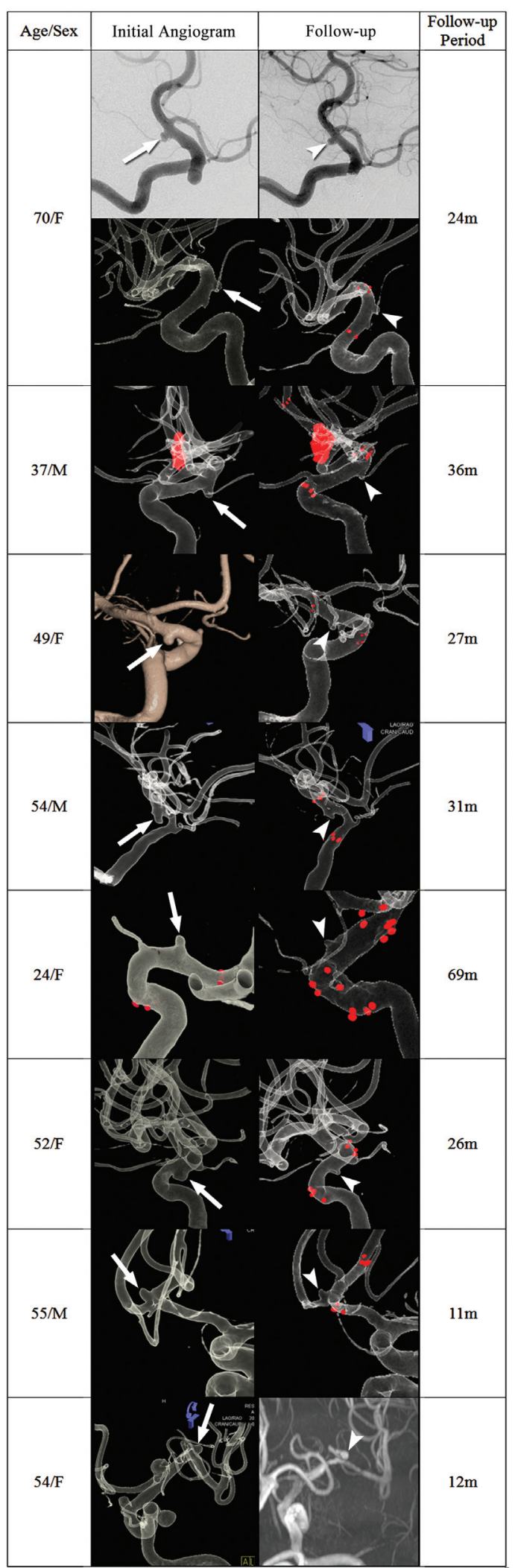

Fig. 1. Comparison of initial and follow-up angiograms for nine aneurysms. Arrows indicate pre-stent aneurysms and arrowheads indicate final post-stent aneurysms on follow-up angiogram.

Note: $-m=$ months, listed number of each patient corresponds to the patient list in Table 1. 
Hemodynamic Effect of Low-wall Coverage Stent in Small Aneurysms

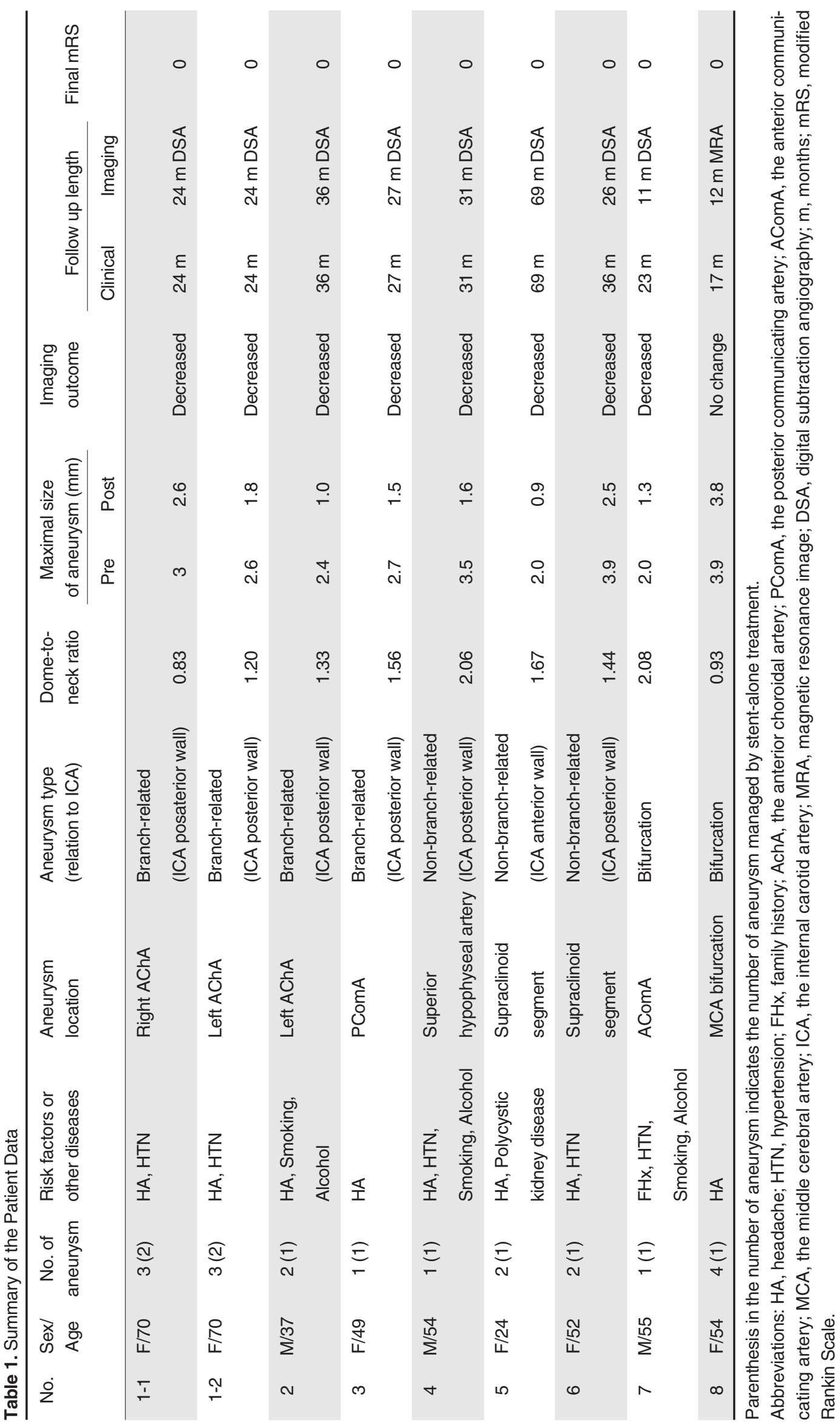




\section{Hairi Liu, et al.}

was established on the basis of digital subtraction angiography (DSA), including 3D rotational angiography [7]. Five patients exhibited multiple aneurysms, either with two $(n=3)$, three $(n=1)$, or four $(n=1)$ aneurysms. Seven of nineteen aneurysms were treated by coiling $(n=4)$ or stent-assisted coiling $(n=3)$. Eight patients with nine aneurysms underwent stenting for the additional aneurysm in patients with multiple aneurysms after treatment of the main target aneurysm $(n=5)$ or as the primary treatment option for patients with single aneurysm according to the rationale describes above $(n=3)$.

Seven aneurysms were located either at the anterior or posterior wall of the supraclinoid or terminal segment of the internal carotid artery (ICA; $n=7$; three at the origin of the anterior choroidal artery [AChA], two at the supraclinoid segment, one at the superior hypophyseal artery and one at the posterior communicating artery $[\mathrm{PComA}])$. And the others were located in the middle cerebral artery bifurcation $(n=1)$ or the anterior communicating artery $(n=1)$. There were 4 branch-related and 3 non-branch-related aneurysms (Table 1).

A 6F guiding catheter was introduced into the cervical segment of the ICA. Diagnostic angiography and 3D DSA were performed using a biplane neuroangiography system (Axiom Artis, Siemens AG, Munich, Germany). A microcatheter was navigated using digital roadmap guidance through the guiding catheter into the targeted artery to cover the lesion. Neuroform (Boston Scientific Corp, Fremont, CA; $\mathrm{n}=8$ ) or Enterprise stent (Codman Neurovascular, Miami, FL; $\mathrm{n}=1$ ) were deployed except for one aneurysm at the dorsal wall of the ICA that was treated with two Neuroform stents.

Patients received clopidogrel ( $75 \mathrm{mg}$ daily) at least 4 days before the procedure and treatment continued for 3-6 months. Patients also received aspirin (100 mg daily) for a year. All endovascular procedures were performed under general anesthesia with systemic heparinization. A bolus of 1500-2500 IU heparin was introduced intravenously at the beginning of each procedure and a 500-1000 IU bolus was administered thereafter every hour according to the activated coagulation time levels. Periprocedural events or complications, such as thromboembolism, dissection, stent migration, aneurysm rupture, or the development of any other neurological symptoms, were evaluated. Angiographic follow-up examinations were performed after the treatment. We evaluated the outcome of the flow-diverting effect of stenting in aneurysms by objective measure and comparison of size of the aneurysm in the maximal length on a working projection (AP, lateral and oblique views, and 3D-angiography) of an initial and follow up angiogram and also by subjective visual assessment of size change of the aneurysm between the initial and follow up angiogram. Clinical outcomes were evaluated during follow-up examinations using the modified Rankin Scale (mRS).

\section{RESULTS}

Clinical data and angiographic follow-up data are summarized (Table 1). No periprocedural complications, including thromboembolism, aneurysm rupture, or stent movement, were observed in any patients. Angiographs obtained immediately after stent deployment showed no obvious changes in aneurysmal morphology when compared with preoperative angiographs. Angiographic follow-up data (median, 31.9 months; range, 17-69 months) were obtained for nine aneurysms in eight patients by 3D DSA $(n=7)$ or MRA $(n=1)$. Eight aneurysms decreased in size and one (patient No. 8) showed no significant interval change in size (Fig. 1 and Table 1). A patient with familial polycystic kidney revealed a new aneurysm at the right AChA origin at 4.5 years follow-up angiography that was not covered by previous stent-assisted coil embolization.

All eight patients scored 0 on the mRS at 6 months. No thrombosis, stenosis, or occlusion of the parent artery related to the stented aneurysms was observed during the mean 31.9-month clinical follow-up period (range, 17-69). At the final clinical evaluation, eight patients scored mRS 0 .

\section{DISCUSSION}

The present study revealed that small aneurysms decreased in size after stenting in most cases. No ruptures or increases in size were observed during the follow-up period. Although a variable degree of aneurysm size decrease may not prevent further growth or rupture of small aneurysms, stenting with a low-wall coverage ratio may have some advantageous hemodynamic effect.

In addition to aneurysm size-dependent hemodynamic effects, the anatomical configuration of the aneurysm in relation to the parent artery may also influence the effect of stenting, as demonstrated in our study. Size criteria, location, multiplicity, family history, presence of symptoms, hypertension and smoking history need to be considered when evaluating 


\section{Hemodynamic Effect of Low-wall Coverage Stent in Small Aneurysms}

the risk of rupture $[8,9]$. Laplace's law states that smaller aneurysms require higher intra-aneurysmal pressure for rupture; thus, smaller aneurysms are associated with more extensive subarachnoid hemorrhage and poor clinical outcomes [10].

There is no known data on the relationship between aneurysm or neck size vs. the stent wall coverage ratio or stent porosity regarding the outcomes of stenting. The stent used in the present study had an $8-10 \%$ wall coverage ratio $(9.5 \%$ for Neuroform and $8.2 \%$ for Enterprise) [11-13]. Although closed cell stents may provide better hemodynamic effects than open cell stents, open cell stents may conform to a curved vessel wall better than a closed cell stent. Therefore, parent artery configuration or the presence of branches related to the aneurysm, as well as aneurysm size, should be considered when selecting the stent.

This study has several limitations. First, the number of patients in the study was small, and data were retrospectively analyzed, although derived from a prospectively collected database. Second, treatment outcomes for small UIAs were obtained by DSA or MRA. However, MRA is also reliable for detecting aneurysm recurrence after stent-assisted coil embolization [14]. Third, aneurysms that decreased in size were not monitored until complete resolution. Although no clinical events, including rupture or other stent-related problems, were observed during the median 19-month follow-up period, further long-term follow-ups of the residual aneurysm are required.

\section{CONCLUSION}

Although a variable degree of aneurysm size decrease may not prevent further growth or rupture of small aneurysms, stenting with a low-wall coverage ratio may have some advantageous hemodynamic effect. Flow modification of stent architecture vs. aneurysm characteristics, including size and location, on long-term outcome, requires further clarification.

\section{References}

1. Tremmel M, Xiang J, Natarajan SK, Hopkins LN, Siddiqui AH, Levy EI, et al. Alteration of intra-aneurysmal hemodynamics for flow diversion using enterprise and vision stents. World Neurosurg 2010;74:306-315

2. Lee JI, Ko JK, Lee TH, Choi CH, Lee SW, Cho WH. Sole stenting technique for the treatment of uncoilable very small aneurysms in the intracranial internal carotid artery. Neurol Med Chir (Tokyo) 2013;53:310-317

3. Lu J, Liu JC, Wang LJ, Qi P, Wang DM. Tiny intracranial aneurysms: endovascular treatment by coil embolisation or sole stent deployment. Eur J Radiol 2012;81:1276-1281

4. Seshadhri S, Janiga G, Beuing O, Skalej M, Thevenin D. Impact of stents and flow diverters on hemodynamics in idealized aneurysm models. J Biomech Eng 2011;133:071005

5. Kulcsar Z, Wetzel SG, Augsburger L, Gruber A, Wanke I, Rufenacht DA. Effect of flow diversion treatment on very small ruptured aneurysms. Neurosurgery 2010;67:789-793

6. Augsburger L, Farhat M, Reymond P, Fonck E, Kulcsar Z, Stergiopulos N, et al. Effect of flow diverter porosity on intraaneurysmal blood flow. Klin Neuroradiol 2009;19:204-214

7. Jeong YG, Kim EH, Hwang SM, Lee GY, Kim JW, Choi YJ, et al. Outpatient (Same-day care) Neuroangiography and Neurointervention. Neurointervention 2012;7:17-22

8. Sonobe M, Yamazaki T, Yonekura M, Kikuchi H. Small unruptured intracranial aneurysm verification study: SUAVe study, Japan. Stroke 2010;41:1969-1977

9. Jeon JS, Ahn JH, Huh W, Son YJ, Bang JS, Kang HS, et al. A retrospective analysis on the natural history of incidental small paraclinoid unruptured aneurysm. J Neurol Neurosurg Psychiatry 2014;85:289-294

10. Taylor CL, Steele D, Kopitnik TA Jr, Samson DS, Purdy PD. Outcome after subarachnoid hemorrhage from a very small aneurysm: a case-control series. J Neurosurg 2004;100:623-625

11. Li C, Li Y, Jiang C, Wu Z, Wang Y, Yang X. Stent alone treatment for dissections and dissecting aneurysms involving the basilar artery. J Neurointerv Surg 2015;7:50-55

12. Santillan A, Greenberg E, Patsalides A, Salvaggio K, Riina HA, Gobin YP. Long-term clinical and angiographic results of Neuroform stent-assisted coil embolization in wide-necked intracranial aneurysms. Neurosurgery 2012;70:1232-1237

13. Fargen KM, Hoh BL, Welch BG, Pride GL, Lanzino G, Boulos $\mathrm{AS}$, et al. Long-term results of enterprise stent-assisted coiling of cerebral aneurysms. Neurosurgery 2012;71:239-244

14. Cho YD, Kim KM, Lee WJ, Sohn CH, Kang HS, Kim JE, et al. Time-of-flight magnetic resonance angiography for follow-up of coil embolization with enterprise stent for intracranial aneurysm: usefulness of source images. Korean J Radiol 2014;15:161-168 